\title{
Numerical Solution of a Two-Class LWR Traffic Flow Model by High-Resolution Central-Upwind Scheme
}

\author{
Jianzhong Chen ${ }^{1}$, Zhongke $\mathrm{Shi}^{1}$, and Yanmei $\mathrm{Hu}^{2}$ \\ ${ }^{1}$ College of Automation, Northwestern Polytechnical University, Xi'an, Shaanxi \\ 710072, P.R. China \\ jzhchen1976@126.com, zkeshi@nwpu.edu.cn \\ ${ }^{2}$ College of Science, Chang'an University, Xi'an, Shaanxi 710064, P.R. China \\ yanmeihu@126.com
}

\begin{abstract}
A high-resolution semi-discrete central-upwind scheme for solving a two class Lighthill-Whitham-Richards (LWR) traffic flow model is investigated in this paper. This scheme is based on combining a fourthorder central weighted essentially nonoscillatory (CWENO) reconstruction with semi-discrete central-upwind numerical flux. The CWENO re construction is chosen to improve the accuracy and guarantee the non-oscillatory behavior of the present method. The strong stability preserving Runge-Kutta method is used for time integration. The resulting method is applied to simulating several tests such as mixture of the two traffic flows. The simulated results illustrate the effectiveness of the present method.
\end{abstract}

Keywords: Traffic flow model, central-upwind scheme, CWENO reconstruction.

\section{Introduction}

Continuum traffic flow models are of great practical importance in many applications such as traffic simulation, traffic control, and, etc. The Lighthill-WhithamRichards (LWR) model proposed independently by Lighthill and Whitham [1] and Richards [2] is the forerunner for all other continuum traffic flow models. In recent years an amount of research was done in implementing and extending the LWR model. Zhang [3] and Jiang et al. 4] proposed higher-order continuum models. Wong and Wong [5] presented a multi-class LWR traffic flow model(MCLWR model). For numerical method, the Lax-Friedrichs scheme was used to solve the MCLWR model in [5]. The Lax-Friedrichs scheme is only first-order accurate and yields a relatively poor resolution due to the excessive numerical dissipation. Recently, the Godunov scheme was also employed to solve the LWR model 6] and higher-order model 77. However, the Godunov scheme needs to use exact or approximate Riemann solvers, which make the scheme complicated and time-consuming. Zhang, et al. [8] pointed out that the scalar LWR model and those higher-order continuum models proposed so far contain hyperbolic partial differential equations. One important feature of this type equation is that it 
admits both smooth and discontinuous solutions such as shocks. However, the lower order numerical methods may produce smeared solutions near discontinuities due to excessive numerical viscosity. The high-order scheme can provide the satisfactory resolution. Moreover, the problems in which solutions contain rich smooth region structures can be resolved by the high-order scheme on a relatively small number of grid points. To embody traffic phenomena described by traffic flow model completely and resolve discontinuities well, a high-resolution shockcapturing numerical method is required. A recent application of the weighted essentially non-oscillatory (WENO) scheme can be found in 89.

In this paper we study another type shock-capturing scheme, the so-called high-resolution semi-discrete central-upwind schemes originally introduced in [10, which have attracted considerable attention more recently. These schemes enjoy the advantages of high-resolution central schemes. They are free of Riemann solvers, require no characteristic decompositions and retain high resolution similar to the upwind results. At the same time, they have an upwind nature. These features make the semi-discrete central-upwind schemes a universal, efficient and robust tool for a wide variety of applications. With regard to its application to traffic flow problems, we have not yet seen any research works. In this work the semi-discrete central-upwind scheme combined with fourth-order central WENO (CWENO) reconstruction [1] is applied to a two class LWR traffic flow model.

This paper is organized as follows. Section 2 presents the two class LWR traffic flow model. In section 3 we describe our numerical method. Numerical simulations are carried out in section 4 . The conclusions are given in section 5 .

\section{The Tow-Class Model}

The MCLWR model 5 describes the characteristics of traffic flow of $M$ classes of road users with different speed choice behaviors in response to the same density when traveling on a highway section. There are some difficulties to compute the eigenvalues and prove the hyperbolicity of the model for $M>3$. In this paper, we consider the two-class $(M=2)$ LWR traffic flow model, which can be written in conservation form as

$$
\mathbf{u}_{t}+\mathbf{f}(\mathbf{u})_{x}=0
$$

where $\mathbf{u}$ is the vector of conserved variables and $\mathbf{f}(\mathbf{u})$ is the vector of fluxes. These are given respectively by

$$
\mathbf{u}=\left[\begin{array}{l}
\rho_{1} \\
\rho_{2}
\end{array}\right], \mathbf{f}(\mathbf{u})=\left[\begin{array}{l}
\rho_{1} u_{1}(\rho) \\
\rho_{2} u_{2}(\rho)
\end{array}\right],
$$

where $\rho_{1}$ and $\rho_{2}$ are the densities for Class 1 and Class 2 traffic, respectively, $\rho=\rho_{1}+\rho_{2}$ is the total density, and $u_{1}(\rho)$ and $u_{2}(\rho)$ are the velocity-density relationships. The two eigenvalues of the Jacobian are

$$
\lambda_{1,2}=\left(u_{1}(\rho)+\rho_{1} u_{1}^{\prime}(\rho)+u_{2}(\rho)+\rho_{2} u_{2}^{\prime}(\rho) \pm \sqrt{\Delta}\right) / 2,
$$


where

$$
\Delta=\left(\left(u_{1}(\rho)+\rho_{1} u_{1}^{\prime}(\rho)\right)-\left(u_{2}(\rho)+\rho_{2} u_{2}^{\prime}(\rho)\right)\right)^{2}+4 \rho_{1} \rho_{2} u_{1}^{\prime}(\rho) u_{2}^{\prime}(\rho) .
$$

Since $\Delta \geq 0$ and $\lambda_{1,2}$ are real, the model is hyperbolic.

\section{Numerical Scheme}

For simplicity, let us consider a uniform grid, $x_{\alpha}=\alpha \Delta x, t^{n}=n \Delta t$, where $\Delta x$ and $\Delta t$ are the uniform spatial and time step, respectively. The cell average in the spatial cell $I_{j}=\left[x_{j-1 / 2}, x_{j+1 / 2}\right]$ at time $t=t^{n}$ is denoted by $\mathbf{u}_{j}^{n}(t)=\frac{1}{\Delta x} \int_{I_{j}} \mathbf{u}\left(x, t^{n}\right) \mathrm{d} x$. Starting with the given cell averages $\left\{\mathbf{u}_{j}^{n}\right\}$, a piecewise polynomial interpolant is reconstructed

$$
\tilde{\mathbf{u}}(x)=\sum_{j} p_{j}^{n}(x) \chi_{j}(x) .
$$

Here $\chi_{j}$ is the characteristic function of the interval $I_{j}$ and $p_{j}^{n}(x)$ is a polynomial of a suitable degree. Different semi-discrete central-upwind schemes will be characteristic of different reconstructions. Given such a reconstruction, the point-values of $\tilde{\mathbf{u}}$ at the interface points $\left\{x_{j+1 / 2}\right\}$ are denoted by $\mathbf{u}_{j+1 / 2}^{+}=$ $p_{j+1}^{n}\left(x_{j+1 / 2}, t^{n}\right)$ and $\mathbf{u}_{j+1 / 2}^{-}=p_{j}^{n}\left(x_{j+1 / 2}, t^{n}\right)$. The discontinuities of the construction (44) at the cell interfaces propagate with right- and left-sided local speeds, which can be estimated by

$$
\begin{aligned}
& a_{j+1 / 2}^{+}=\max \left\{\lambda_{N}\left(\frac{\partial \mathbf{f}}{\partial \mathbf{u}}\left(\mathbf{u}_{j+1 / 2}^{-}\right)\right), \lambda_{N}\left(\frac{\partial \mathbf{f}}{\partial \mathbf{u}}\left(\mathbf{u}_{j+1 / 2}^{+}\right)\right), 0\right\} \\
& a_{j+1 / 2}^{-}=\min \left\{\lambda_{1}\left(\frac{\partial \mathbf{f}}{\partial \mathbf{u}}\left(\mathbf{u}_{j+1 / 2}^{-}\right)\right), \lambda_{1}\left(\frac{\partial \mathbf{f}}{\partial \mathbf{u}}\left(\mathbf{u}_{j+1 / 2}^{+}\right)\right), 0\right\} .
\end{aligned}
$$

Here $\lambda_{1}, \cdots, \lambda_{N}$ denote the $N$ eigenvalues of $\partial \mathbf{f} / \partial \mathbf{u}$. The semi-discrete centralupwind scheme for the spatial discretization of equation (11) can be given by(see [10] for the detailed derivation)

$$
\frac{d}{d t} \mathbf{u}_{j}(t)=-\frac{H_{j+1 / 2}(t)-H_{j-1 / 2}(t)}{\Delta x},
$$

where the numerical fluxes $H_{j+1 / 2}$ is

$$
\begin{aligned}
H_{j+1 / 2}(t)= & \frac{a_{j+1 / 2}^{+} \mathbf{f}\left(\mathbf{u}_{j+1 / 2}^{-}\right)-a_{j+1 / 2}^{-} \mathbf{f}\left(\mathbf{u}_{j+1 / 2}^{+}\right)}{a_{j+1 / 2}^{+}-a_{j+1 / 2}^{-}} \\
& +\frac{a_{j+1 / 2}^{+} a_{j+1 / 2}^{-}}{a_{j+1 / 2}^{+}-a_{j+1 / 2}^{-}}\left[\mathbf{u}_{j+1 / 2}^{+}-\mathbf{u}_{j+1 / 2}^{-}\right] .
\end{aligned}
$$

Note that different semi-discrete central-upwind schemes are typical of different reconstructions. The accuracy of the semi-discrete scheme (6)-(7) depends 
on the accuracy of the reconstruction (44). One can use the second order piecewise linear reconstruction, the third-order piecewise quadratic reconstruction, highly accurate essentially non-oscillatory (ENO) reconstruction, highly accurate WENO reconstruction or highly accurate CWENO reconstruction. In this work, we have used an fourth-order CWENO reconstruction proposed in [1] to compute the point values $\mathbf{u}_{j+1 / 2}^{ \pm}$. To simplify notations, the superscript $n$ will be omitted below. In each cell $I_{j}$, the reconstruction, $p_{j}(x)$, is a convex combination of three quadratic polynomials, $q_{l}(x), l=j-1, j, j+1$,

$$
p_{j}(x)=\omega_{j-1}^{j} q_{j-1}(x)+\omega_{j}^{j} q_{j}(x)+\omega_{j+1}^{j} q_{j+1}(x),
$$

where $\omega_{l}^{j}$ are the weights which satisfy $\omega_{l}^{j} \geq 0$ and $\sum_{l=j-1}^{j+1} \omega_{l}^{j}=1$. The quadratic polynomials $q_{l}(x), l=j-1, j, j+1$, are given by

$$
q_{l}(x)=\tilde{\mathbf{u}}_{l}+\tilde{\mathbf{u}}_{l}^{\prime}\left(x-x_{l}\right)+\tilde{\mathbf{u}}_{l}^{\prime \prime}\left(x-x_{l}\right)^{2}, l=j-1, j, j+1 .
$$

Here $\tilde{\mathbf{u}}_{l}^{\prime \prime}=\frac{\mathbf{u}_{l+1}-2 \mathbf{u}_{l}+\mathbf{u}_{l-1}}{\Delta x^{2}}, \tilde{\mathbf{u}}_{l}^{\prime}=\frac{\mathbf{u}_{l+1}+\mathbf{u}_{l-1}}{2 \Delta x}$ and $\tilde{\mathbf{u}}_{l}=\mathbf{u}_{l}-\frac{\tilde{\mathbf{u}}_{l}^{\prime \prime}}{24}$. The weights $\omega_{l}^{j}$ are defined as

$$
\omega_{l}^{j}=\frac{\alpha_{l}^{j}}{\alpha_{j-1}^{j}+\alpha_{j}^{j}+\alpha_{j+1}^{j}}, \alpha_{l}^{j}=\frac{C_{l}}{\left(\epsilon+I S_{l}^{j}\right)^{2}}, l=j-1, j, j+1,
$$

where $C_{j-1}=C_{j+1}=3 / 16, C_{j}=5 / 8$. The constant $\epsilon$ is used to prevent the denominators from becoming zero and is taken as $\epsilon=10^{-6}$. The smoothness indicators, $I S_{l}^{j}$, are calculated by

$$
\begin{aligned}
& I S_{j-1}^{j}=\frac{13}{12}\left(\mathbf{u}_{j-2}-2 \mathbf{u}_{j-1}+\mathbf{u}_{j}\right)^{2}+\frac{1}{4}\left(\mathbf{u}_{j-2}-4 \mathbf{u}_{j-1}+3 \mathbf{u}_{j}\right)^{2}, \\
& I S_{j}^{j}=\frac{13}{12}\left(\mathbf{u}_{j-1}-2 \mathbf{u}_{j}+\mathbf{u}_{j+1}\right)^{2}+\frac{1}{4}\left(\mathbf{u}_{j-1}-\mathbf{u}_{j+1}\right)^{2}, \\
& I S_{j+1}^{j}=\frac{13}{12}\left(\mathbf{u}_{j}-2 \mathbf{u}_{j+1}+\mathbf{u}_{j+2}\right)^{2}+\frac{1}{4}\left(3 \mathbf{u}_{j}-4 \mathbf{u}_{j+1}+\mathbf{u}_{j+2}\right)^{2} .
\end{aligned}
$$

The time discretization of the semi-discrete scheme is achieved by third-order strong stability preserving Runge-Kutta solver [12].

\section{Numerical Examples}

In this section, we choose several numerical examples presented in 9] as out test case. The results demonstrate the performance of the present method for the two-class LWR traffic flow model. In all examples, the following velocity-density relationships are chose:

$$
u_{1}(\rho)=u_{1 f}\left(1-\rho / \rho_{m}\right), u_{2}(\rho)=u_{2 f}\left(1-\rho / \rho_{m}\right),
$$

where $\rho_{m}$ is maximal density and $u_{1 f}$ and $u_{2 f}$ are the free flow velocity for Class 1 and Class 2 traffic, respectively. Moreover, the variables of space, time, density 
and velocity are scaled by $L, T, \rho_{m}$ and $u_{f}$, where $L$ is the length of the road, $T$ is computational time and $u_{f}=\max \left(u_{1 f}, u_{2 f}\right)$. A variable is also non-dimensional if it is not followed by its unit.

Example 1: Mixture of the two traffic flows.

The computational parameters are $L=6000 \mathrm{~m}, T=400 \mathrm{~s}, \Delta x=60 \mathrm{~m}, \Delta t=$ $0.4 \mathrm{~s}, u_{1 f}=14 \mathrm{~m} / \mathrm{s}$ and $u_{2 f}=20 \mathrm{~m} / \mathrm{s}$. The initial data is taken as the following Riemann problem:

$$
\mathbf{u}(x, 0)= \begin{cases}(0,0.4), & x<0.1 \\ (0.4,0), & x>0.1\end{cases}
$$

In this test Class 2 traffic will mix in Class 1 traffic, which causes the increase of total density. Its solution contains a shock, a constant region and a rarefaction. The total density computed by the presented method is shown in Fig. 1. To illustrate the advantage of using high-order schemes, the Godunov scheme with the Rusanov approximate Riemann solver [1314] is also adopted to compute the same problem using the same parameters. Here and below, this scheme is abbreviated to GR. The scheme presented in this paper is abbreviated to CP4. The result computed by GR scheme is presented in Fig. 2. This comparison demonstrates the clear advantage of SD4 scheme over GR scheme. The SD4 scheme has the higher shock resolution and smaller numerical dissipation.

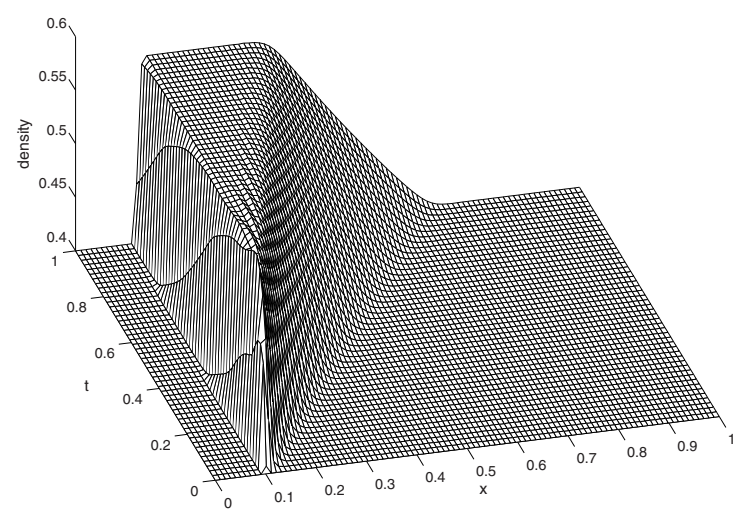

Fig. 1. Example 1: The total density. The solution is computed with CP4 scheme.

Example 2: Separation of the two traffic flows.

The parameters are $L=8000 \mathrm{~m}, T=400 \mathrm{~s}, \Delta x=80 \mathrm{~m}, \Delta t=0.4 \mathrm{~s}, u_{1 f}=10 \mathrm{~m} / \mathrm{s}$ and $u_{2 f}=20 \mathrm{~m} / \mathrm{s}$. The Riemann initial data is used:

$$
\mathbf{u}(x, 0)= \begin{cases}(0.2,0), & x<0.1 \\ (0,0.2), & x>0.1\end{cases}
$$




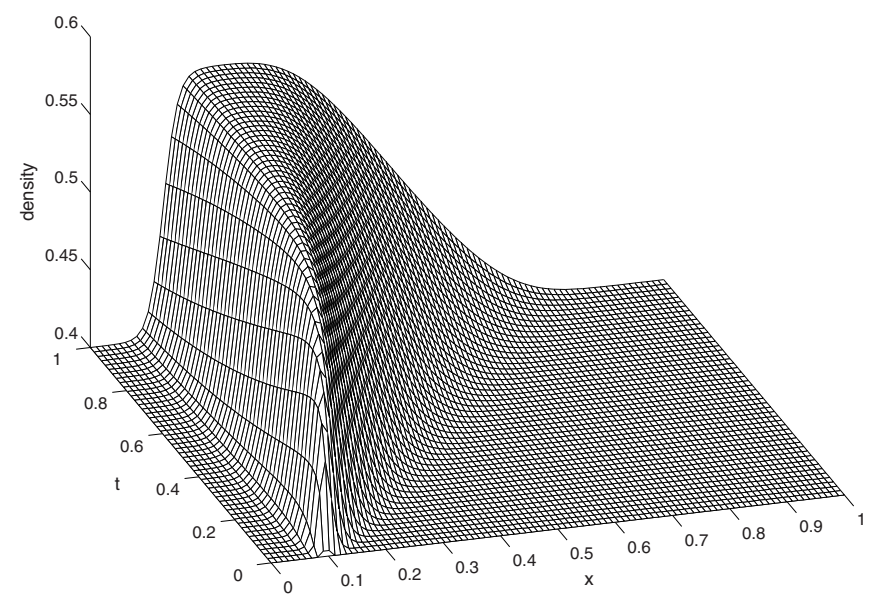

Fig. 2. Example 1: The total density. The solution is computed with GR scheme.

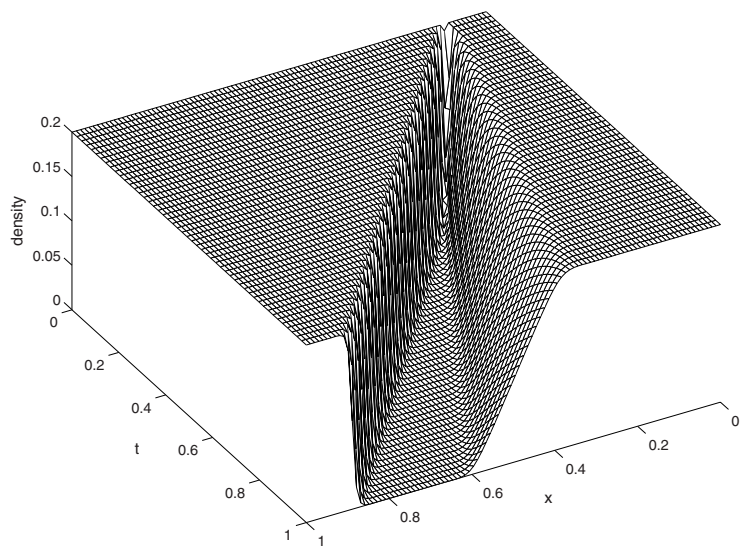

Fig. 3. Example 2: The total density. The solution is computed with CP4 scheme.

Note that $u_{2}=16 \mathrm{~m} / \mathrm{s}>u_{1 f}$ and thus Class 1 drivers can not keep up with Class 2 drivers. A vacuum region is formed between Class 1 and Class 2 traffic. This test has solution consisting of a right shock, a constant region and a left rarefaction. Figs. 3 and 4 show the results obtained with CP4 and GR scheme, respectively. It can be seen that discontinuities are well resolved by CP4 scheme.

Example 3: A close following of the two traffic flows.

The parameters are $L=4000 \mathrm{~m}, T=240 \mathrm{~s}, \Delta x=80 \mathrm{~m}, \Delta t=0.4 \mathrm{~s}, u_{1 f}=14 \mathrm{~m} / \mathrm{s}$ and $u_{2 f}=20 \mathrm{~m} / \mathrm{s}$. The Riemann initial data is used:

$$
\mathbf{u}(x, 0)=\left\{\begin{array}{lc}
(0.2,0), & x<0.1, \\
(0,0.44), & x>0.1 .
\end{array}\right.
$$

The high resolution properties of CP4 scheme are illustrated in Fig. 5. 


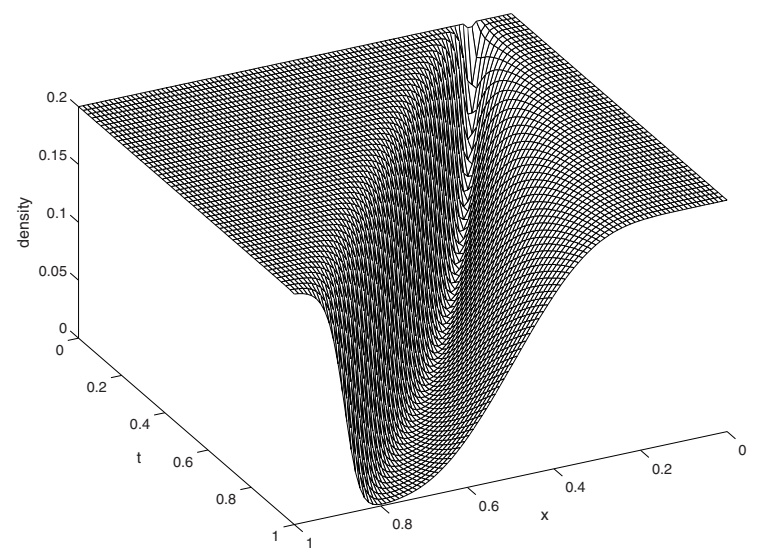

Fig. 4. Example 2: The total density. The solution is computed with GR scheme.

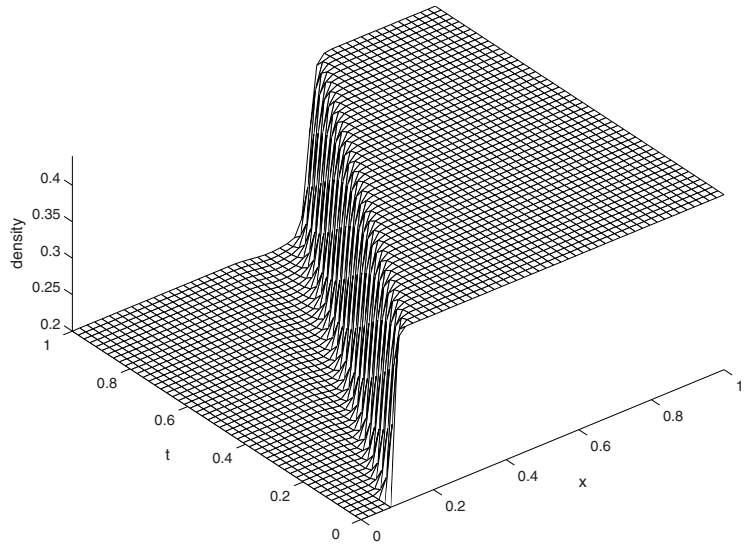

Fig. 5. Example 3: The total density. The solution is computed with CP4 scheme.

\section{Conclusions}

As an attempt to simulate traffic flow by high-resolution finite difference schemes, we have applied the semi-discrete central-upwind scheme to a two class LWR traffic flow model in this paper. The numerical results demonstrate that the semidiscrete central-upwind scheme resolve the shock and rarefaction waves well. This universal, efficient and high-resolution scheme will be implemented and applied to higher-order continuum models and multi-class models to simulate traffic flow in our future work. 


\section{References}

1. Lighthill, M. J., Whitham, G. B.: On kinematic waves (II)-A theory of traffic flow on long crowed roads. Proc. R. Sco. London, Ser. A 22 (1955) 317-345

2. Richards, P. I.: Shock waves on the highway. Oper. Res. 4 (1956) 42-51

3. Zhang, H. M.: A non-equilibrium traffic model devoid of gas-like behavior. Transportation Research B 36 (2002) 275-290

4. Jiang, R., Wu, Q. S., Zhu, Z. J.: A new continuum model for traffic flow and numerical tests. Transportation Research B 36 (2002) 405-419

5. Wong, G. C. K., Wong, S. C.: A multi-class traffic flow model-an extension of LWR model with heterogeneous drivers. Transportation Research A 36 (2002) 827-841

6. Lebacque, J. P.: The Godunov scheme and what it means for first order traffic flow models. In: Lesort, J. B. (eds.): Proceedings of the 13th International Symposium on Transportation and Traffic Theory. Elsevier Science Ltd., Lyon France (1996) 647-677

7. Zhang, H. M.: A finite difference approximation of a non-equilibrium traffic flow model. Transportation Research B 35 (2001) 337-365

8. Zhang, M. P., Shu, C.-W., Wong, G. C. K., Wong, S. C.: A weighted essentially nonoscillatory numerical scheme for a multi-class Lighthill-Whitham-Richards traffic flow model. Journal of Computational Physics 191 (2003) 639-659

9. Zhang, P., Liu, R. X., Dai, S. Q.: Theoretical analysis and numerical simulation on a two-phase traffic flow LWR model. Journal of university of science and technology of China 35 (2005) 1-11

10. Kurganov, A., Noelle, S., Petrova, G.: Semi-discrete central-upwind schemes for hyperbolic conservation laws and Hamilton-Jacobi equations. SIAM J. Sci. Comput. 23 (2001) 707-740

11. Levy, D., Puppo, G., Russo, G.: Central WENO schemes for hyperbolic systems of conservation laws. Math. Model. Numer. Anal. 33 (1999) 547-571

12. Gottlieb, S., Shu, C.-W., Tadmor, E.: Strong stability preserving high order time discretization methods. SIAM Rev. 43 (2001) 89-112

13. Toro, E. F.: Riemann Solvers and Numerical Methods for Fluid Dynamics. Springer-Verlag, Berlin Heidelberg New York (1997)

14. Rusanov, V. V.: Calculation of interaction of non-steady shock waves with obstacles. J. Comput. Math. Phys. 1 (1961) 267-279 